\title{
THE AUTOMATA COMPLEXITY \\ OF THE LANGUAGE TRANSFORMATION SCHEMA THAT CONTAINS OPERATIONS WITH RESTRICTED DEGREE
}

\author{
NGUYEN VAN DINH
}

\begin{abstract}
In accordance with the concept of automaton with the output we have built the language transformation schema $\Sigma$ (see [6]). In this paper we study the relation between the automata complexity $g(\Sigma)$, the number of essential vertices $|\Sigma|$ and the depth of operations $s$ on a language transformation schema. The estimation of the automata complexity of a language transformation schema that holds operations with restricted degree is also given.
\end{abstract}

Tóm tắt. Dựa trên khái niệm ô tô mát có lối ra, ta xây dựng được lược đồ biến đổi ngôn ngũ (BĐNN) [6]. Khi các lược đồ BĐNN chứa các phép lặp không hạn chế thì độ phức tạp ô tô mát của nó không phụ thuộc vào số phép lặp và đã được đánh giá bởi [6], nhưng khi số phép lặp, cũng như một số phép toán khác như phép lấy các cặp tù chăn, lấy các cặp từ lẻ, phép bổ sung..., có bậc bị hạn chế thì độ phưc tạp ô tô mát cưa các lược đồ BĐNN sẽ phụ thuộc vào độ sâu đặt dấu (bậc) cưa các phép toán.

Bài này trình bày mối liên hệ giữa độ phưc tạp ô tô mát, số đỉnh cốt yếu và độ sâu đặt dấu các phép toán của một lược đồ BĐNN có chứa các phép toán có bậc được hạn chế.

\section{INTRODUCTION}

Using notation $\mathcal{L}=\mathcal{X} \times \mathcal{Y}$, wherein $\mathcal{X}=\left\{x_{1}, x_{2}, \ldots, x_{n}\right\}$ is input alphabet of source (original) language, and $\mathcal{Y}=\left\{y_{1}, y_{2}, \ldots, y_{m}\right\}$ is output alphabet of target (final) language. An automaton with the output, $M=\left(S, \mathcal{X}, \mathcal{Y}, s_{0}, \delta, \lambda, F\right)$, recognizes a language on the input alphabet, symbolized $T_{X}$, and transforms it into another language on the output alphabet, that is symbolized $T_{Y}$. This language transformation is due to automata mappings, on which the lengths of words are completely preserved. (See [6]). In brief, the automaton $M$ recognizes a language pair $R=\left(T_{X}, T_{Y}\right)$. Corresponding to automaton with the output $M$, we can build the language transformation schema equivalent to $M$, which also recognizes the same language pair with the initial automaton. (See [6]). When the language transformation schema holds unrestricted repetitions, its automata complexity does not depend on the number of the repetitions and has been appraised by [6]. However, if the number of the repetitions, as well as another operations: creating even word pairs, odd word pairs or complement... have limited degrees, the automata complexity of the language transformation schema will be reliant on the depth of operations. This paper is to analyze evaluate the automata complexity of a language transformation schema that contains operations with restricted degree.

\section{PRELIMINARY CONCEPTS}

Some preliminary concepts about the language transformation schema presented in [6] are summarized in this part. In addition, new relating concepts will be separately described. The language pair $\left(T_{X}, T_{Y}\right)$ on the dual alphabet $\mathcal{L}=\chi \times \mathcal{Y}$, denoted $R$, and $R^{m}$ is pair of repeated language with degree $m$ of $T_{X}$ and $T_{Y}$. The subset of $R$, contains word pairs with either even or odd lengths, denoted $\mathcal{E}(R)$ or $\mathcal{O}(R)$, respectively. The set of all initial parts of word pairs, of which lengths are at most $s$, denoted $\mathcal{H}(R, s)$.

The language transformation graph on the dual alphabet $\mathcal{L}=\mathcal{X} \times \mathcal{Y}$ is a directed graph $G$, in which, a particular vertex called entry vertex, denoted $\sigma(G)$, and a nonempty set of vertices called the set of final vertices, denoted $F(G)$, and we denote the set of all vertices as $A(G)$. Initial vertex and 
final vertex of an edge $a$ are denoted $\alpha(a)$ and $\beta(a)$, respectively. On each edge $a$ of the graph $G$ is labelled by a group of word pairs on the dual alphabet $\mathcal{L}$, this group is denoted $M_{G}(a)$. Suppose that every edge $a_{1}, a_{2}, \ldots, a_{t}(t \geq 1)$ is a certain path in $G$ and $T_{i} \in M_{G}\left(a_{i}\right)$ is word pairs corresponding to edge $a_{i}(1 \leq i \leq t)$, then word pairs $T_{1} T_{2} \ldots T_{t}$ are considered is created by this path. We denote the set of all word pairs generated by path initiating from vertex $\alpha$ and ending at vertex $\beta$ as $N_{G}(\alpha, \beta)$. The set $N_{G}(\alpha, \beta)$ is inductively defined as the following principles:

1. $\Lambda=(\varepsilon, \varepsilon)$, wherein $\varepsilon$ is an empty word of $\chi^{*}$ and $y^{*}$.

2. If $X, Y$ are word pairs, wherein $X \in N_{G}(\alpha, \gamma)$, and $\gamma=\alpha(a), \beta=\beta(a), Y \in M_{G}(a)$ then $X Y \in N_{G}(\alpha, \beta)$.

Definition 2.1. The language transformation schema $\Sigma$ on the dual alphabet $\mathcal{L}=\mathcal{X} \times \mathcal{Y}$ is a range of the language transformation graphs on $\mathcal{L}$ :

$$
\Sigma=\left(G_{1}, G_{2}, \ldots, G_{n}\right)
$$

and on this range, we build a function $m_{\Sigma}(a)$, defined on the set of all edges of all graphs $G_{i}$ $(1 \leq i \leq n)$ and satisfied the following conditions:

1. For a certain edge a of $G_{i}(1 \leq i \leq n)$, the function $m_{\Sigma}(a)$ is satisfied one of following standards:

1.1. $m_{\Sigma}(a)=\Lambda$ and $M_{G_{i}}(a)=\Lambda$, then $a$ is called an empty edge.

1.2. $m_{\Sigma}(a)=(x, y) \in \mathcal{L}$ and $M_{G_{i}}(a)=\{x, y\}$, then edge $a$ notes a pair of symbols $(x, y)$ and edge $a$ is called essential edge.

1.3. $m_{\Sigma}(a)=G_{j}(1 \leq j \leq n-1)$ and $M_{G_{i}}(a)=\mathcal{E}\left(N\left(G_{j}\right)\right)$, then edge $a$ is stated to depending on graph $G_{j}$ and is called an even edge.

1.4. $m_{\Sigma}(a)=G_{k}(1 \leq k \leq n-1)$ and $M_{G_{i}}(a)=\mathcal{O}\left(N\left(G_{k}\right)\right)$, then edge $a$ is stated to depending on graph $G_{k}$ and is called an odd edge.

1.5. $m_{\Sigma}(a)=G_{r}(1 \leq r \leq n-1)$ and $M_{G_{i}}(a)=N^{S}\left(G_{r}\right)$, then edge $a$ is stated to depending on graph $G_{r}$ and is called repeated edge with degree $s$.

1.6. $m_{\Sigma}(a)=G_{t}(1 \leq t \leq n-1)$ and $M_{G_{i}}(a)=\mathcal{H}\left(N\left(G_{t}\right), s\right)$, then edge $a$ is stated to depending on graph $G_{t}$ and it is called complement edge with degree $s$.

2. Each graph $G_{1}, G_{2}, \ldots, G_{n-1}$ has one and only one edge of graph $G_{n}$ depending on. Graph $G_{n}$ called a base graph.

3. Graphs $G_{1}, G_{2}, \ldots, G_{n}$ have no common vertex.

$G_{n}$ is called the base graph of the language transformation schema. If $\Sigma$ contains only one graph, this unique graph is also signed $\Sigma$, and called a simple language transformation schema. The set of word pairs $N\left(G_{n}\right)$ is considered to be created by $\Sigma$, and denoted $N(\Sigma)$. We at times use $N_{X}(\Sigma)$ and $N_{Y}(\Sigma)$ to denote separately the set of origin and the final words defined by $\Sigma$. Obviously, $N(\Sigma) \subseteq N_{X}(\Sigma) \times N_{Y}(\Sigma)$.

The vertex $\alpha$ of graph $G$ is called an essential vertex if it is entered by at least one essential edge. The number of essential vertices of graph $G$ is signed $|G|$. The number of essential vertices of all graph belong to $\Sigma$ is signed $|\Sigma|$. We state that graph $G_{i}$ depends on graph $G_{j}$ if it contains some edge depending on graph $G_{j}$.

Definition 2.2. For the language transformation schema, the depth of operations, denoted $l(\Sigma)$, and determined as follow:

Let $a$ is an unintentional edge of graph $G_{r}(1 \leq r \leq n)$, then the depth of operations of $a$ is signed $l(a)$, and we define the depth of operation in $G$ by the formula:

wherein $l(a)$ is inductively determined as follows:

$$
l(G)=\max _{a \in G} l(a)
$$

1. If $a$ is an even edge, odd edge, repeated edge with degree $n$ or complement edge with degree $s$ and $a$ depends on graph $G_{j}(1 \leq j \leq i)$, and $l\left(G_{j}\right)$ has been defined, then:

$$
l(a)=l\left(G_{j}\right)+1 \text {. }
$$


2. For the remaining cases, $l(a)=0$.

The equation $l(\Sigma)=l\left(G_{n}\right)$ is admitted.

Definition 2.3. Possible minimum number of states of an weak deterministic finite automaton with output (see [6]) which recognizes $N(\Sigma)$, is called the automata complexity of the language transformation schema $\Sigma$, and denoted $g(\Sigma)$.

\section{THE RESULTS}

In this part, we will prove a theorem so that to appraise the automata complexity $g(\Sigma)$ of the language transformation schema $\Sigma$ which depends on the number of essential vertices $|\Sigma|$ and the depth of operation $s$.

Firstly, we prove some lemmas.

Lemma 3.1. For every simple language transformation schema $\Sigma$, there exists an weak deterministic finite automaton with the output $A$, such that:

$$
T(A)=N(\Sigma) \text { and }|A| \leq 2^{|\Sigma|}+1 .
$$

Proof. As $\Sigma$ is a simple language transform schema, thus, according to [6], it is possible to build an automaton with output, $M=\left(S, \mathcal{X}, \mathcal{Y}, s_{0}, \delta, \lambda, F\right)$ as follorv:

- $S$ : the set of states of automaton $M$, includes all vertex signs of $\Sigma$.

- Entry vertex sign of $\Sigma$ is regarded as initiating state $s_{0}$ of automaton $M$.

- The set of final vertices of $\Sigma$ is admitted to be a set of final states of $M$.

- State transitional function $\delta: S \times \Sigma \rightarrow S$ of automaton $M$ is determined: $\forall s \in S, \forall x \in \mathcal{X}$ then $\delta(s, x)=\left\{s_{i 1}, s_{i 2}, \ldots, s_{i t}\right\} \Leftrightarrow \forall j$ with $1 \leq j \leq t$ then: $x \in N_{X}\left(s, s_{i j}\right), s_{i j} \in S$.

- The output function $\lambda: S \times \mathcal{X} \rightarrow \mathcal{Y}$ of automaton $M$ is determined: $\forall s \in S, \forall x \in \mathcal{X}$ then $\lambda(s, x)=y \in \mathcal{Y}$, wherein $y$ is an element corresponding to $x$ in the pair $(x, y)$ which is written in the edge $(s, \delta(s, x))$ of the simple language transformation schema $\Sigma$.

- The input and output alphabet $\mathcal{X}, \mathcal{Y}$ of the simple language transformation schema $\Sigma$ are considered as the input and output alphabet, respectively, of automaton $M$.

In this way, it is obviously that $T_{X}(M)=N_{X}(\Sigma)$ and $T_{Y}(M)=N_{Y}(\Sigma)$. Indeed, we have build an automaton $M$ that recognizes the same language pair with the simple language transformation schema $\Sigma$.

With automaton $M$, using algorithm and determinazing automata program [7], we can build an weak deterministic finite automaton with output $A$ that equates to $M$ (i.e. automata $A$ recognizes the same set of word pairs with automaton $M)$. In addition, as the results in [2], then:

$$
T(A)=T(M)=N(\Sigma) \text { and }|A| \leq 2^{|M|}+1=2^{|\Sigma|}+1 .
$$

Thus, the lemma is proved.

Lemma 3.2. For every simple language transformation schema $\Sigma$, there exists another simple schema $\Sigma^{\prime}$, such that:

$$
N\left(\Sigma^{\prime}\right)=\mathcal{E}(N(\Sigma)) \text { and }\left|\Sigma^{\prime}\right| \leq 2|\Sigma| \text {. }
$$

Proof. 1. Builds schema $\Sigma_{0}$ : This schema includes two vertices $\alpha_{0}$ and $\alpha_{1}$. Vertex $\alpha_{0}$ is entry as well as final vertex of $\Sigma_{0}$. From $\alpha_{0}$ to $\alpha_{1}$ and conversely from $\alpha_{1}$ to $\alpha_{0}$, there are just right $n$ edges on each of which, one of symbols from the dual alphabet $\mathcal{L}=\chi x \times \mathcal{Y}$ is written, and two different edges are written with two different pairs of symbols. Thus, the schema $\Sigma_{0}$ just right two essential vertices, i.e. $\left|\Sigma_{0}\right|=2$, and $N\left(\Sigma_{0}\right)$ contains word pairs whose lengths are even.

2. Build schema $\Sigma^{\prime}$ : We regard $\Sigma^{\prime}$ as an intersection of schemas $\Sigma_{0}$ and $\Sigma$, as $\Sigma^{\prime}$ is the intersection of simple schemas, according to the results of $[6]$, the conclusion is:

$$
N\left(\Sigma^{\prime}\right)=N\left(\Sigma_{0}\right) \cap N(\Sigma) \text { and }\left|\Sigma^{\prime}\right| \leq\left|\Sigma_{0}\right| \cdot|\Sigma|=2 \cdot|\Sigma| .
$$

Obviously, $N\left(\Sigma^{\prime}\right)$ is a set of word pairs possessing even lengths. 
The lemma has been proved.

Lemma 3.3. For every simple language transformation schema $\Sigma$, there exists another simple schema $\Sigma^{\prime}$, such that:

$$
N\left(\Sigma^{\prime}\right)=\mathcal{O}(N(\Sigma)) \text { and }\left|\Sigma^{\prime}\right| \leq 2|\Sigma| \text {. }
$$

Proof. 1. Builds a schema $\Sigma_{1}$ : This schema is structured the same as $\Sigma_{0}$, except $\alpha_{0}$ is input vertex, whereas $\alpha_{1}$ is unique final vertex. From $\alpha_{0}$ to $\alpha_{1}$ and conversely from $\alpha_{1}$ to $\alpha_{0}$, there are just right $n$ edges, on each of which one pair of symbols from the dual alphabet $\mathcal{L}=\mathcal{X} \times \mathcal{Y}$ is written, and two different edges are written with two different pairs of symbols. Hence, the schema $\Sigma_{1}$ has just right two essential vertices, obviously, $\left|\Sigma_{1}\right|=2$, and $N\left(\Sigma_{1}\right)$ contains word pair whose lengths are odd only.

2. Build up a schema $\Sigma^{\prime}$ : We regard $\Sigma^{\prime}$ as an intersection of schemas $\Sigma_{1}$ and $\Sigma$, as $\Sigma^{\prime}$ is the intersection of simple schemas, according to the results of [6], the conclusion is:

$$
N\left(\Sigma^{\prime}\right)=N\left(\Sigma_{1}\right) \cap N(\Sigma) \text { and }\left|\Sigma^{\prime}\right| \leq\left|\Sigma_{1}\right| \cdot|\Sigma|=2 \cdot|\Sigma| .
$$

On the other hand, obviously $N\left(\Sigma^{\prime}\right)$ is a set of word pairs possessing odd lengths.

The lemma has been proved.

Lemma 3.4. For every simple language transformation schema $\Sigma$ and with any integer $s>0$, there exists another simple schema $\Sigma^{\prime}$, such that:

$$
N\left(\Sigma^{\prime}\right)=N_{s}^{*}(\Sigma) \text { and }\left|\Sigma^{\prime}\right| \leq s|\Sigma| .
$$

Proof. Build up a schema $\Sigma^{\prime}$ : Put from left to right a range of language transformation schemas that are structured as same as schema $\Sigma$. Nevertheless, their vertices are symbolized variously. Each final vertex of $i^{\text {th }}$-schema $(0 \leq i \leq s-1)$ has an empty edge linking with the entry vertex of $i+1^{\text {th }}$-schema.

The entry vertex of the first schema is considered as the entry of $\Sigma^{\prime}$, and the set of final vertex of $s^{\text {th }}$-schema become the final vertex of $\Sigma^{\prime}$. Obviously:

$$
N\left(\Sigma^{\prime}\right)=N_{s}^{*}(\Sigma) \text { and }\left|\Sigma^{\prime}\right| \leq s \cdot|\Sigma| \text {. }
$$

The lemma is proved.

Lemma 3.5. For every simple language transformation schema $\Sigma$ and with any integer $m>0$, there exists another simple schema $\Sigma^{\prime}$, such that:

$$
N\left(\Sigma^{\prime}\right)=\mathcal{H}(N(\Sigma), m) \text { và }\left|\Sigma^{\prime}\right| \leq m \cdot|\Sigma| .
$$

Proof. 1. Build up a schema $\Sigma_{m}$, including $m+1$ vertices: $\alpha_{0}, \alpha_{1}, \ldots, \alpha_{m-1}, \alpha_{m}$. From $\alpha_{i}$ to $\alpha_{i+1}$ $(0 \leq i \leq m-1)$ there are $n$ edges on which different pairs of signs extracted from the dual alphabet $\mathcal{L}$ are written. From $\alpha_{m-1}$ to $\alpha_{m}$, there are $n$ edges on which different pairs of signs from $\mathcal{L}$ are written. The vertex $\alpha_{0}$ is regarded as the entry, and $\alpha_{0}, \alpha_{1}, \ldots, \alpha_{m-2}, \alpha_{m-1}$ are acknowledged as final vertices of $\Sigma_{m}$. Thus, schema $\Sigma_{m}$ recognizes all possible word pairs on the dual alphabet $\mathcal{L}$, whose lengths are bounded at $\mathrm{m}$ and they have just right $m$ essential vertices.

2. Build up a schema $\Sigma^{\prime}$ as an intersection of $\Sigma_{m}$ and $\Sigma$, of which the pair of vertices containing final vertex of $\Sigma_{m}$ is recognized as final vertex of $\Sigma^{\prime}$. It is clearly that $\Sigma^{\prime}$ is a simple schema, and according to the results of [6], the conclusion is: $N\left(\Sigma^{\prime}\right)=\mathcal{H}(N(\Sigma), m)$, this is a set of all first parts of word pairs whose lengths are at most $m$, and $\left|\Sigma^{\prime}\right| \leq m .|\Sigma|$.

The lemma is proved.

Lemma 3.6. For any the language transformation schema $\Sigma$, of which the depth of operations restricted at $s$, there exists a simple schema $\Sigma^{\prime}$, such that:

$$
N\left(\Sigma^{\prime}\right)=N(\Sigma) \text { and }\left|\Sigma^{\prime}\right| \leq s^{l(\Sigma)} \cdot|\Sigma| \text {. }
$$

Proof. The lemma is proved by mathematical induction on to the depth of operations on the schema $\Sigma$.

1. If $\Sigma$ is a simple schema, obviously, we can regard $\Sigma^{\prime}$ as $\Sigma$, therefore:

$$
N\left(\Sigma^{\prime}\right)=N(\Sigma) \text { and }\left|\Sigma^{\prime}\right|=|\Sigma|=s^{0} \cdot|\Sigma|=s^{l(\Sigma)} \cdot|\Sigma| .
$$


2. Suppose that, $\Sigma=\left(G_{1}, G_{2}, \ldots, G_{m-1}\right)$ and $a_{1}, a_{2}, \ldots, a_{p}$ are even edges, odd edges or complement edges with degree $t$, repeated edges with degree $u$ on the graph $G_{n}$. As the depth of operations not exceed $s$, thus $t \leq s$ and $u \leq s$.

Suppose that, $a_{i}(1 \leq i \leq p)$ depends on the graph $G_{k i}$. For every $i(1 \leq i \leq p)$ it is possible to use all the graphs on which $G_{k i}$ depends, including $G_{k i}$, to build schema $\Sigma_{k i}$. Then we have:

$$
N\left(\Sigma_{k i}\right)=N\left(G_{k i}\right) \text {. }
$$

According to the definition of the depth of operations, there is a conclusion:

$$
l(\Sigma)=l\left(G_{n}\right) \geq l\left(a_{i}\right)+1=l\left(G_{k i}\right)+1=l\left(\Sigma_{k i}\right)+1 .
$$

Hence:

$$
l\left(\Sigma_{k i}\right) \leq l(\Sigma)-1 .
$$

To match the definition of induction, for each $i(1 \leq i \leq p)$, there exists a simple schema $\Sigma_{k i}^{\prime}$, such that:

$$
N\left(\Sigma_{k i}^{\prime}\right)=N\left(\Sigma_{k i}\right) \text { and }\left|\Sigma_{k i}^{\prime}\right| \leq\left|\Sigma_{k i}\right| . s^{l\left(\Sigma_{k i}\right)} \leq\left|\Sigma_{k i}\right| \cdot s^{l(\Sigma)-1} .
$$

If $a_{i}$ is an even edge (or an odd edge), then, in accordance with Lemma 3.2 (or Lemma 3.3) we can build up a simple schema $\Delta_{i}$, such that:

$$
\begin{gathered}
N\left(\Delta_{i}\right)=\mathcal{E}\left(N\left(\Sigma_{k i}^{\prime}\right)\right)=\mathcal{E}\left(N\left(\Sigma_{k i}\right)\right)=\mathcal{E}\left(N\left(G_{k i}\right)\right) \\
\text { (or } N\left(\Delta_{i}\right)=\mathcal{O}\left(N\left(\Sigma_{k i}^{\prime}\right)\right)=\mathcal{O}\left(N\left(\Sigma_{k i}\right)\right)=\mathcal{O}\left(N\left(G_{k i}\right)\right), \text { respectively) }
\end{gathered}
$$

with:

$$
\left|\Delta_{i}\right| \leq 2\left|\Sigma_{k i}^{\prime}\right| \leq 2\left|\Sigma_{k i}\right| \cdot s^{l(\Sigma)-1} \leq\left|\Sigma_{k i}\right| \cdot s^{l(\Sigma)} .
$$

If $a_{i}(1 \leq i \leq p)$ is the complement edge with degree $r$ (or repeated edge with degree $r$ ), then according to Lemma 3.5 (or Lemma 3.4) we can build a simple schema $\Delta_{i}$, such that:

$$
\begin{gathered}
N\left(\Delta_{i}\right)=\mathcal{H}\left(N\left(\Sigma_{k i}^{\prime}\right), r\right)=\mathcal{H}\left(N\left(\Sigma_{k i}\right), r\right)=\mathcal{H}\left(N\left(G_{k i}\right), r\right) \\
\text { (or } N\left(\Delta_{i}\right)=N_{r}^{*}\left(\Sigma_{k i}^{\prime}\right)=N_{r}^{*}\left(\Sigma_{k i}\right)=N_{r}^{*}\left(G_{k i}\right), \text { respectively) }
\end{gathered}
$$

with:

$$
\left|\Delta_{i}\right| \leq r \cdot\left|\Sigma_{k i}^{\prime}\right| \leq r .\left|\Sigma_{k i}\right| s^{l(\Sigma)-1} \leq\left|\Sigma_{k i}\right| s^{l(\Sigma)} \text {. }
$$

Replace $a_{i}(1 \leq i \leq p)$ on $G_{n}$ with schema $\Delta_{i}$, in accordance with the definitions of substitution (see[6]), we have a simple schema $\Sigma^{\prime}$, such that:

$$
N\left(\Sigma^{\prime}\right)=N(\Sigma)
$$

with:

$$
\begin{aligned}
\left|\Sigma^{\prime}\right| & =\left|G_{n}\right|+\sum_{1 \leq i \leq p}\left|\Delta_{i}\right| \\
& \leq\left|G_{n}\right|+\sum_{1 \leq i \leq p}\left|\Sigma_{k i}\right| s^{l(\Sigma)} \\
& \leq\left|G_{n}\right| s^{l(\Sigma)}+\sum_{1 \leq i \leq p}\left|\Sigma_{q i}\right| s^{l(\Sigma)} \\
& =\left(\left|G_{n}\right|+\sum_{1 \leq i \leq p}\left|\Sigma_{k i}\right|\right) s^{l(\Sigma)} \\
& =|\Sigma| s^{l(\Sigma)} .
\end{aligned}
$$

The lemma is proved.

Theorem. For any language transformation schema $\Sigma$, on which the depth of operations restricted at $s$, then:

$$
g(\Sigma) \leq 2^{|\Sigma| s^{l(\Sigma)}}+1 .
$$

Proof. 1. For the language transformation schema $\Sigma$ as above, using Lemma 3.6, we can build up a simple schema $\Sigma^{\prime}$, such that:

$$
N\left(\Sigma^{\prime}\right)=N(\Sigma) \text { and }\left|\Sigma^{\prime}\right| \leq|\Sigma| s^{l(\Sigma)} .
$$

2. For this simple schema $\Sigma^{\prime}$, using Lemma 3.1, we can build up an weak deterministic finite automaton with output $A$, such that: 


$$
\begin{gathered}
T(A)=N\left(\Sigma^{\prime}\right)=N(\Sigma), \text { and: } \\
g(\Sigma)=|A| \leq 2^{\left|\Sigma^{\prime}\right|}+1 \leq 2^{|\Sigma| s^{l(\Sigma)}}+1 .
\end{gathered}
$$

The theorem has been proved.

\section{REFERENCES}

[1] Arto Saloma., Computation and Automata (in Vietnamese), Science Publishers, 1992.

[2] Dang Huy Ruan, O сложности конечного автомата, соответствую щего обобщенному регулиарному выражению, ДАН СССР, Том 213, No. 1 (1973).

[3] Dominique Perrin, Finite Automata, J. van Leeuwen (ed.), Handbook of Theorytical Computer Science, Elsevier Science Publishers B.V., 1990, p. 3-53.

[4] J. E. Hopcroft and J. D. Ullman, Introduction to Automata Theory, Languages and Computation, Addison-Wesley, Reading, MA, 1979.

[5] Lung H., "An Algebraic method for Solving decision problems in Finite Automata theory", Ph.D. Thesis, Penn. State Univ. University Park, PA, 1987.

[6] Nguyen Van Dinh, "Builds the language transformation schema and analyses its automata complexity", Master Thesis, Vietnam National University (VNU), Hanoi, 1997.

[7] Nguyen van Dinh, Solving determinazation problems of automata on the computer, VNU Journal of Science, Nat. Sci., XIV (1) (1998).

[8] Peterson J.L., Petri nets, Computing Surveys 9 (3) (1997).

United Nations International School-Hanoi

Received August 20, 2000

Revised January 10, 2001 